International Journal of Zoological Investigations

Contents available at Journals Home Page: www.ijzi.net

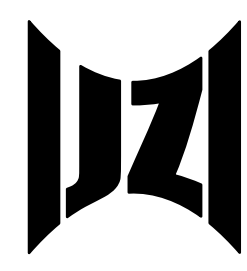

ISSN: 2454-3055

\title{
In Vitro Study of Effect of Low Temperature Treatment on Contraction of Colon and Rectum of Adult and Neonate Rats
}

\author{
Singh Shuchita* and Mandal Maloy B. \\ Department: Department of Physiology, Institute: Institute of Medical Science, \\ Banaras Hindu University, Varanasi 221005, India \\ *Corresponding Author
}

Received: $13^{\text {th }}$ October, 2020

Accepted: $9^{\text {th }}$ November, 2020

Published online: $10^{\text {th }}$ November, 2020

https://doi.org/10.33745/ijzi.2020.v06i02.012

\begin{abstract}
Temperature is an important environmental factor which affects the physiology of different system of body including gut. Many studies in different species showed that temperature affects not only contractile tension or tone of gut smooth muscle but also its frequency of contractions. Therefore, present study aims to assess the effect of low temperature on colon and rectum of neonatal and adult rats and to find out mechanisms involved.

In the present study, lowering bath temperature (from 37-15 C), caused temperature-dependent increase in the contractile tension (g/g wet tissue) and decrease in contractile frequency in both colon and rectum of adult and neonate rat resulting into a direct relation with frequency and inverse relation to the contractile force or tone. Although there was no difference in contractile tension between colon and rectum of adult rats, the same was not true for neonate. The rectum of neonate seemed to be more responsive to temperature change than colon. A temperature of 15-20 C abolished rhythmic activity of gut smooth muscle with heightened tone. This low temperature-induced contractile response were non-cholinergic but involved diltiazem sensitive L-type $\mathrm{Ca}^{2+}$ channel in adult rectum and not in neonate. Such response indicated existence of a differential mechanism in adult and neonate rectum.
\end{abstract}

Keywords: Colon, Rectum, Diltiazem, Calcium channel

Citation: Singh Shuchita and Mandal Maloy B.: In Vitro study of effect of low temperature treatment on contraction of colon and rectum of adult and neonate rats. Intern. J. Zool. Invest. 6 (2): 330-337, 2020. https://doi.org/10.33745/ijzi.2020.v06i02.012

\section{Introduction}

Temperature is an important environmental factor which affects the physiology of different system of body including gut. Studies in rat gastrointestinal tract revealed that lowering the gut temperature altered its contractile pattern (Yano et al., 1982; Takeuchi et al., 
1990; Sabeur, 1996; Mustafa and Thulesius, 2001; Dong et al., 2010). In rat intestine it has been demonstrated that cooling induced tonic contractions. These contractions were inversely proportional to temperature. Decrease in temperature also caused elimination of rhythmic activity of gut smooth muscle and involved calcium translocation ((Mustafa and Thulesius, 2001). In another study, in rat large intestine, it was also reported that decrease in temperature could induce tonic contractions in longitudinal muscle segments of colon while phasic contractions either decreased or disappeared. These work also suggested the role of transient receptor potential A1 (TRPA1) in cold-induced contractions and L- type calcium channel and neurogenic mechanisms were also implicated in cold induced contractions (Dong et al., 2010). Sabeur (1996) reported that acetylcholine-induced contractile in rat small gut was higher at $21 \mathrm{C}$ than at $33 \mathrm{C}$ in a calcium-free medium. Thus, temperature causes change in gut motility and altered contractile activity of gut to low temperature. These changes may be mediated via calcium channel, neurogenic or transient receptor potential mechanisms (Mustafa and Thulesius, 2001; Dong et al., 2010).

Temperature has been reported to affect not only contractile tension or tone of gut smooth muscle but also its frequency of contractions. For example in mice small intestine, direct linear relation between temperature and frequency of contraction has been observed. Therefore, the frequency was seen to decrease with decrease in temperature (Studier et al., 1975).

The above mentioned studies were carried out in adult gut but there is lack of study on neonate gut after change in temperature.
Therefore, present study aims to assess the effect of low temperature on colon and rectum of neonatal and adult rats and to find out mechanisms involved.

\section{Materials and Methods}

Animals:

Adult Albino rats of Charles Foster strain of 46 months and neonate of 10-16 days of same strain were used. The animals were housed in a temperature, humidity and light controlled room (12 h light and $12 \mathrm{~h}$ dark) with an ad libitum supply of food and water. The animal experiments were performed as per guidelines of the Ethical Clearance Committee of the Institute of Medical Sciences, Banaras Hindu University, Varanasi, India.

\section{Dissection of animal:}

Adult rats were killed by cervical dislocation and exsanguinations and neonate by decapitation. The abdomen was opened immediately by vertical incision and part of the gut (ileum) was extirpated and cleaned by flushing out the gut contents and placed in a Petri dish containing chilled Krebs Ringer solution bubbled with $100 \% \mathrm{O}_{2}$.

Mounting and recording of contractile
response: After cleaning, longitudinal segments $(1.5 \mathrm{~cm})$ of colon and rectum were dissected out and placed in an organ bath $(10 \mathrm{ml})$ containing Krebs-Ringer solution maintained at $37 \pm 1 \mathrm{C}$ and continuously bubbled with $100 \% \mathrm{O}_{2}$. The tissue segments were mounted vertically. One end of the tissue was fastened to a glass tube support, and the other end was fixed to a force transducer (MLT 0210, AD Instruments, Sydney, Australia) with an initial tension of 0.25-0.5 g. 
The preparations were allowed to equilibrate for 30 minutes before taking the control recordings. Isometric contractions signals from force transducer were recorded with the help of data acquisition system (Power Lab 4/ST system) displayed through a personal computer. Recorded contractions were analyzed with the help of software Chart-5 for windows (AD Instruments, Sydney, Australia). Before, as well as after recording the contractile responses, calibration for the tension (0-10 g) was performed. After stabilization, the initial recordings of spontaneous contractions were made for $30 \mathrm{~min}$ at $37 \mathrm{C}$. Subsequently, the tissue segment was exposed to different bath temperature (37 C, $30 \mathrm{C}, 25 \mathrm{C}, 20 \mathrm{C}$ and $15 \mathrm{C}$ ). The preparation was subjected to each temperature for $10 \mathrm{~min}$ and contractile responses were recorded in each temperature.

Since, maximum contractile tone was obtained at $20 \mathrm{C}$, therefore, another set of experiments were carried out to understand the mechanism of contraction by using different antagonists then contractions were recorded for colon and rectum of both adult and neonate. The temperature of organ bath was maintained by hot or cold water as required.

After recording of contractions, the segment of tissue was removed from the organ bath and placed on blotting paper for lightly soaking the extra water from the tissue. The two end of the tissue was cut to remove the injured parts. The wet tissue was then weighed in a fine balance to express the contractile response per unit weight of tissue (g/g wet tissue).
Drugs and solutions:

Sodium chloride $(\mathrm{NaCl})$, potassium chloride $(\mathrm{KCl})$, magnesium sulphate $\left(\mathrm{MgSO}_{4}\right)$, calcium chloride $\left(\mathrm{CaCl}_{2}\right)$, sodium bicarbonate (NaHCO3), potassium dihydrogen phosphate $\left(\mathrm{KH}_{2} \mathrm{PO}_{4}\right)$ and glucose were procured from Loba Chemie Pvt. Ltd., Mumbai, India. KrebsRinger solution was prepared with following compositions (in mM/L): $\mathrm{NaCl}, 119 ; \mathrm{KCl}, 4.7$; $\mathrm{CaCl}_{2} .2 \mathrm{H}_{2} \mathrm{O}, 2.5 ; \mathrm{KH}_{2} \mathrm{PO}_{4}, 1.2 ; \mathrm{MgSO}_{4} .7 \mathrm{H}_{2} \mathrm{O}, 1.2$; $\mathrm{NaHCO}_{3}, 5$; and glucose, 11 and $\mathrm{pH}$ of the solution was 7.4 .

Atropine $5 \mathrm{mM}$ stock solution in distilled water, diltiazem $5 \mathrm{mM}$ stock solution in distilled water, nifedipine $5 \mathrm{mM}$ stock solution in absolute alcohol. Atropine and nifedipine were purchased from sigma, and diltiazem from torrent pharmaceuticals, India.

Experimental protocol:

After taking initial recording of contractile response from colon and rectum of both neonate and adult rats ( $n=6$ for each), for 30 minute then tissue was subjected to different bath temperature of $37 \mathrm{C}, 30 \mathrm{C}, 25 \mathrm{C}, 20 \mathrm{C}$ and $15 \mathrm{C}$ to record the temperature-response relation. Same protocol applied for neonate colon and rectum. Then they are exposed to different antagonists at $37 \mathrm{C}$ to find out mechanism of low temperature-induced contractions.

\section{Statistical Analysis:}

The recordings of contractions were saved in the personal computer and were retrieved later. Thereafter, the recordings were further analyzed to express the contractions in the terms of highest tone or contractile tension (g/g wet tissue). 
The mean \pm SE value of contractile tension ( $\mathrm{g} / \mathrm{g}$ wet tissue) in colon and rectum of adult and neonate rat was calculated. The temperature-response relationships within group were compared by two way ANOVA. Student's t-test for paired and unpaired observations was performed. A value of $\mathrm{p}<$ 0.05 was considered as significant.

\section{Results}

Effect of temperature change (37 to 15 C) on contractile tension in colon and rectum of adult rats:

There was a temperature-dependent rise in contractile tension (g/g wet tissue) in both colon and rectum of adult rats. As temperature decreases from 37 to $15 \mathrm{C}$ the contractile tension was found to be increased till $20 \mathrm{C}$. Further, decrease in temperature to $15 \mathrm{C}$ either did not have any effect or there was decrease in the response. However, there was no significant difference of contractile tone between colon and rectum of adult rats ( $p>0.05$, two way ANOVA, Fig. 1).

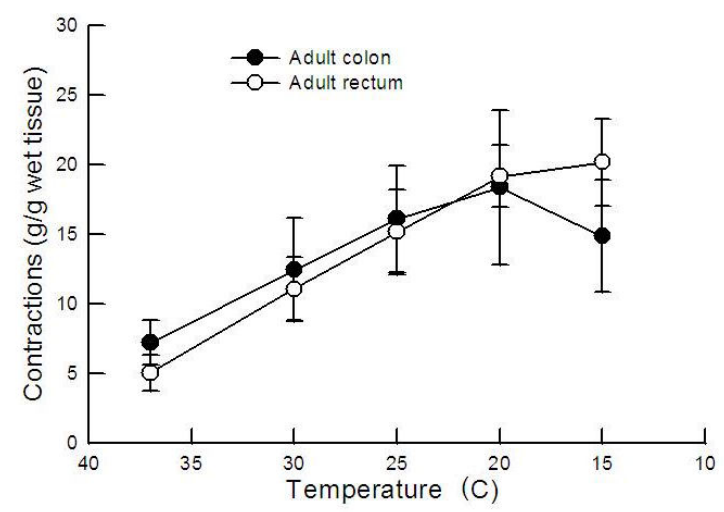

Figure 1: Dose-response curve showing the effect of change in bath temperature $(37 \mathrm{C}, 30 \mathrm{C}, 25 \mathrm{C}, 20 \mathrm{C}$ and $15 \mathrm{C}$ ) on contractile tension (g/g wet tissue) in colon and rectum of adult rats. Decrease in bath temperature increased contractile tension in colon and rectum of adult rats in a temperature-dependent manner but there was no difference in contractile tension between colon and rectum $(p<0.05$, two way ANOVA, $n=6)$. Data points indicate mean \pm SE values.
Effect of temperature change from 37 to $15 \mathrm{C}$ on contractile tension in neonate colon and rectum:

In case of neonate there was also temperature-dependent increase in contractile tension (g/g wet tissue) as temperature decreased from 37 to $15 \mathrm{C}$ in both colon and rectum. Further, increase in contractile tension was significantly more in rectum as compared to colon ( $\mathrm{p}<0.05$, two way ANOVA, Fig. 2).

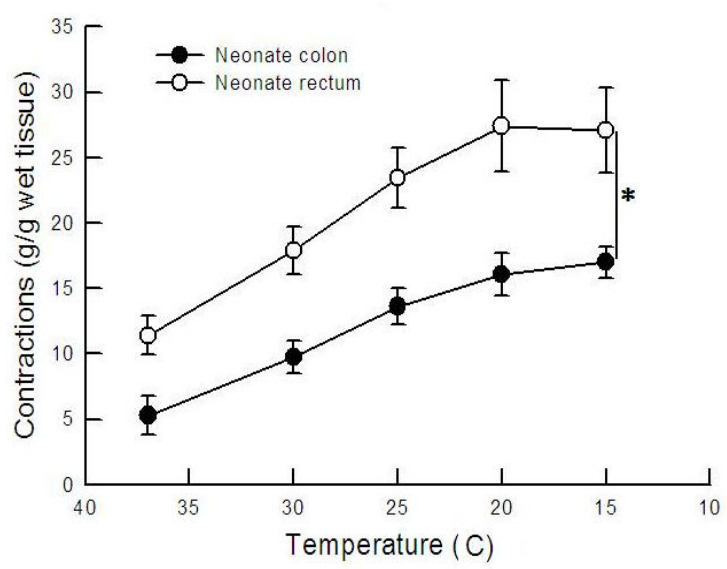

Figure 2: Dose-response curve showing the effect of change in bath temperature $(37 \mathrm{C}$ to $15 \mathrm{C})$ on contractile tension (g/g wet tissue) in colon and rectum of neonate rats. Decrease in bath temperature increased contractile tension in colon and rectum of neonate rats in a temperature-dependent manner and contractile tension was significantly more in rectum than colon $(p<0.05$, two way ANOVA, $n=6)$. Data points indicate mean $\pm \mathrm{SE}$ values.

Effect of temperature change from 37 to $15 \mathrm{C}$ on contractile frequency in colon and rectum of adult and neonate rats:

There was also temperature-dependent decrease in contractile frequency in colon and rectum of both adult and neonate (Figs. 3, 4). As temperature changes from 37 to $15 \mathrm{C}$, frequency of contractions decreases significantly more in colon than its rectum of adult rats $(\mathrm{p}<0.05$, two way ANOVA, $n=6$, Fig. 
3). Whereas in case of neonate there was no significant difference between colon and rectum ( $p>0.05$, two way ANOVA, Fig. 4).

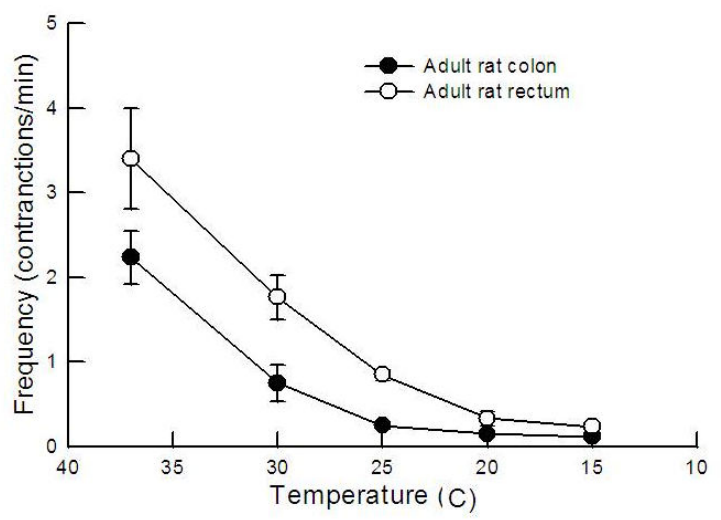

Figure 3: Dose-response curve showing the effect of change in bath temperature (37 C - $15 \mathrm{C}$ ) on contractile frequency in colon and rectum of adult rats. Decrease in bath temperature caused reduction in contractile frequency in colon and rectum of adult rats as compared to $37 \mathrm{C}(\mathrm{p}<0.05$, Student's t-test, paired, $\mathrm{n}=6)$ and contractile frequency was significantly less in colon than rectum $(p<0.05$, two way ANOVA, $n=6)$. Data points indicate mean \pm SE values.

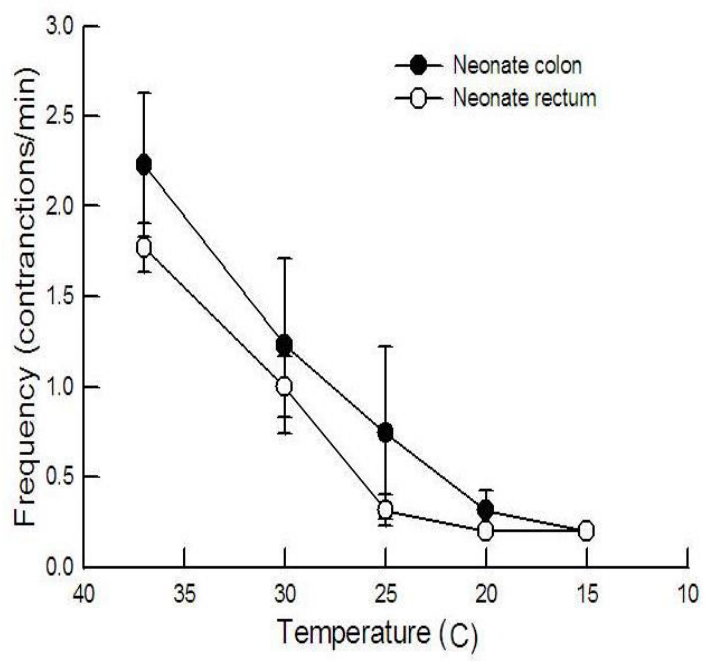

Figure 4: Dose-response curve showing the effect of change in bath temperature (37 C - 15 C) on contractile frequency in colon and rectum of neonate rats. Decrease in bath temperature declined contractile frequency in colon and rectum of neonate rats as compared to $37 \mathrm{C}(\mathrm{p}<0.05$, Student's t-test, paired, $\mathrm{n}=6)$ but there was no difference in contractile frequency between colon and rectum $(\mathrm{p}<0.05$, two way ANOVA, $\mathrm{n}=6$ ). Data points indicate mean \pm SE values.
Effect of antagonists on contractile tension at $20 \mathrm{C}$ :

Response of low temperature is nearly similar at 20 and $15 \mathrm{C}$. Therefore, $20 \mathrm{C}$ has been selected to see the effect of various antagonists on smooth muscle contractions.

Atropine (1 $\mu M)$ failed to inhibit contractile tension at $20 \mathrm{C}$ in both colon and rectum of adult and neonate rats:

Pretreatment with atropine $(1 \mu \mathrm{M})$, a cholinergic antagonist failed to block contractile tension (g/g wet tissue) produced at $20 \mathrm{C}$ in colon and rectum of both adult and neonate (Figs. 5, 6).

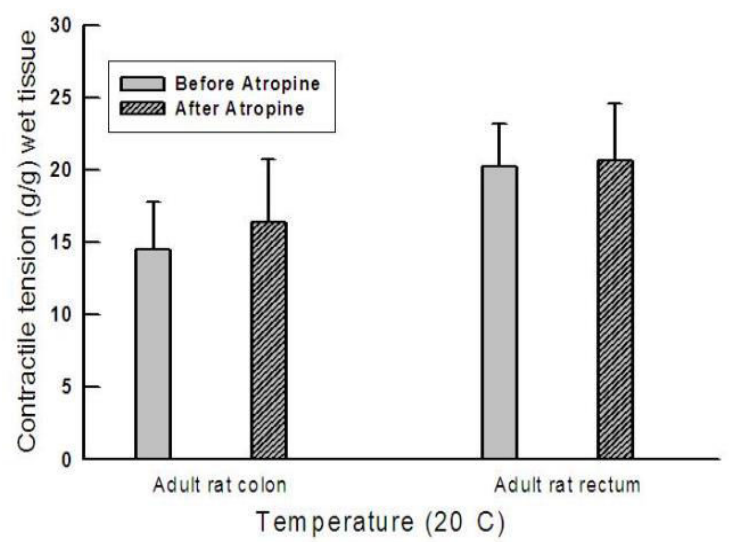

Figure 5: Histograms showing the effect of temperature $(20 \mathrm{C})$-induced contractions before and after atropine $(1 \mu \mathrm{M})$ pretreatment in adult colon and rectum. Atropine pretreatment did not block temperature induced contractile tension in colon and rectum of adult rats $(\mathrm{p}>0.05$, Student's $t$-test, paired, $n=6)$.

Diltiazem (10 $\mu \mathrm{M})$ blocked rise in contractile tension at $20 \mathrm{C}$ in adult rectum only:

Diltiazem (10 $\mu \mathrm{M})$, a L-type calcium channel antagonist, pre-application blocked rise in contractile tension at $20 \mathrm{C}$ in adult rectum but not in its colon. On the other hand, diltiazem failed to block temperature- induced contractile tension in neonate colon and rectum (Figs. 7,8 ). 


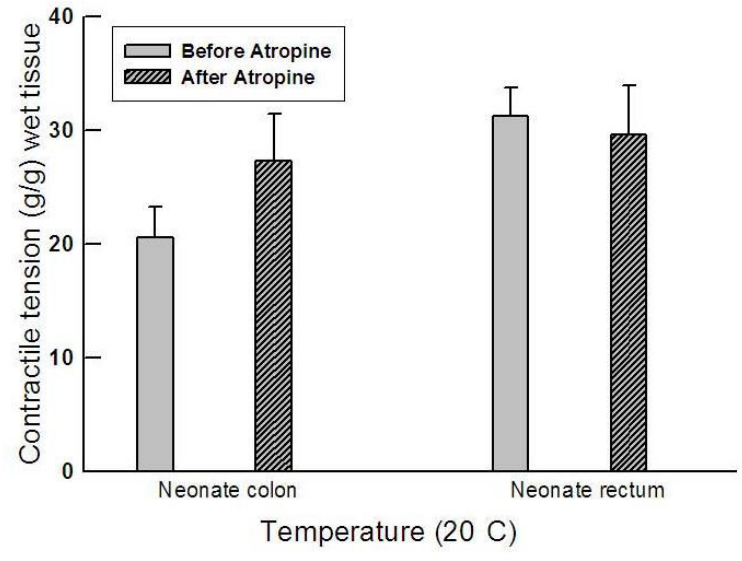

Figure 6: Histograms showing the effect of temperature $(20 \mathrm{C})$ induced contractions before and after atropine $(1 \mu \mathrm{M})$ pretreatment in neonate colon and rectum. Atropine pretreatment failed to block temperature induced contractile tension in colon and rectum of neonate rats ( $p>0.05$, Student's $t$-test, paired, $n=6)$.

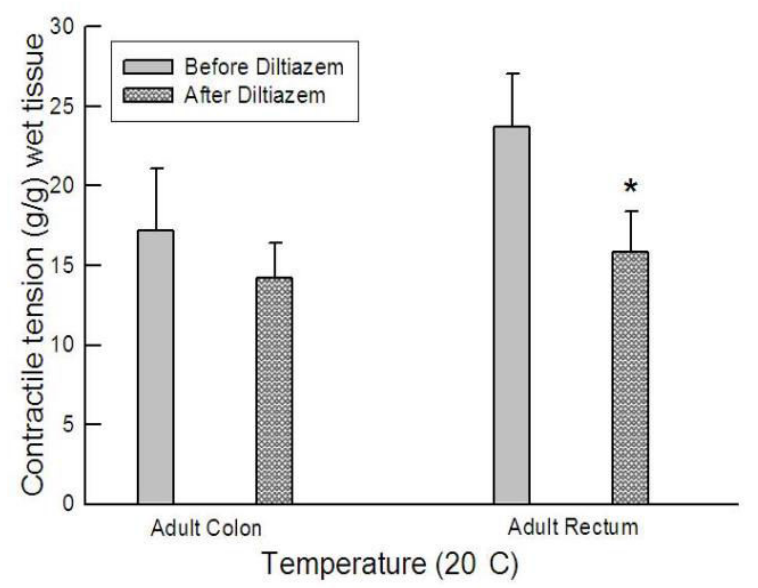

Figure 7: Histograms showing the effect of temperature $(20 \mathrm{C})$ induced contractions before and after diltiazem $(10 \mu \mathrm{M})$ pretreatment in adult colon and rectum. Diltiazem pretreatment significantly blocked temperature -induced increase in contractile tension in adult rectum only ( $\mathrm{p}<0.05$, Student's $t$-test, paired, $n=6)$ and no such response in colon ( $p>0.05$, Student's $t$-test, paired, $\mathrm{n}=6$ ).

Nifedipine $(1 \mu \mathrm{M}$ and $(10 \mu \mathrm{M})$ failed to inhibit contractile tension produced at $20 \mathrm{C}$ in colon and rectum of both adult and neonate rats:

Pretreatment with nifedipine $(1 \mu \mathrm{M})$ and $(10$ $\mu \mathrm{M})$, a L-type calcium channel antagonist, did not block contractile tension at $20 \mathrm{C}$ in both adult as well as in neonate colon and rectum (Figs. 9, 10).

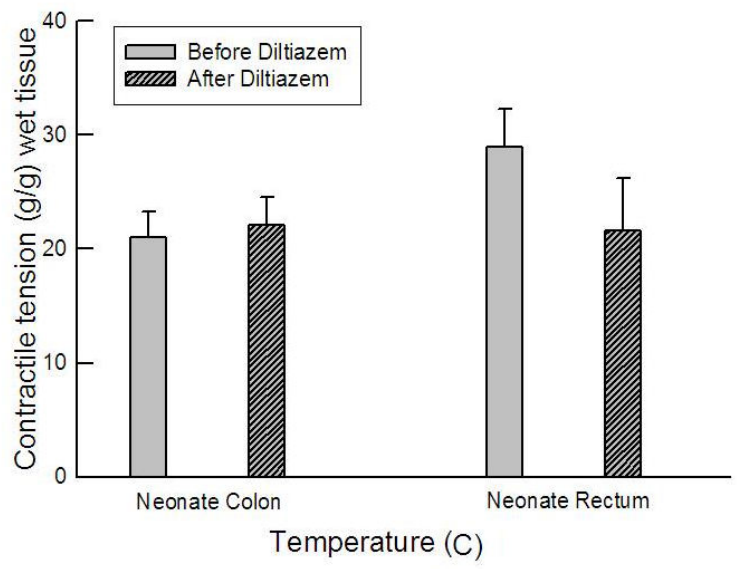

Figure 8: Histograms showing the effect of temperature (20 C) induced contractions before and after diltiazem $(10 \mu \mathrm{M})$ pretreatment in adult colon and rectum. Diltiazem pretreatment did not affect temperatureinduced contractile tension in neonate colon and rectum ( $p>0.05$, Student's $t$-test, paired, $n=6)$.

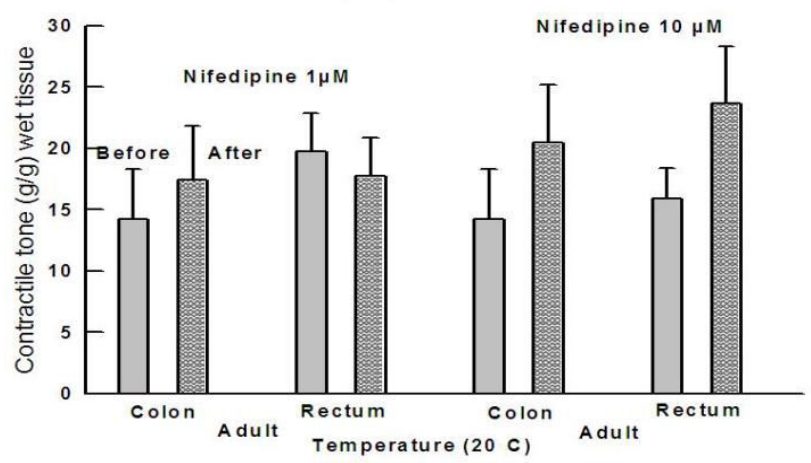

Figure 9: Histograms showing the effect of temperature (20 C) induced contractions before and after Nifedipine $(1 \mu \mathrm{M}$ and $10 \mu \mathrm{M})$ pretreatment in adult colon and rectum. Nifedipine pretreatment did block temperature induced contractile tension in adult colon and rectum. ( $p>0.05$, Student's $t$-test, paired, $n=6$ ).

\section{Discussion}

The present study has demonstrated that there is temperature-dependent increase in the contractile tension (g/g wet tissue) as well as a temperature-dependent decrease in contractile frequency in both colon and rectum of adult and neonate rat. Thus, 


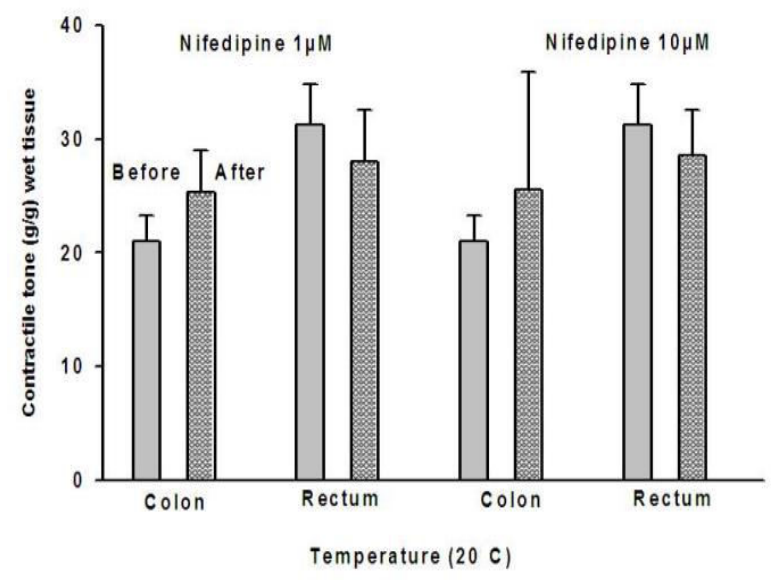

Figure 10: Histograms showing the effect of temperature $(20 \mathrm{C})$ induced contractions before and after Nifedipine $(1 \mu \mathrm{M}$ and $10 \mu \mathrm{M})$ pretreatment in neonate colon and rectum. Nifedipine pretreatment failed to inhibit temperature-induced contractile tension in neonate colon and rectum ( $p>0.05$, Student's $t$-test, paired, $\mathrm{n}=6$ ).

decrease in bath temperature from $37-15 \mathrm{C}$ is inversely proportional to rise in contractile tension and directly proportional to contractile frequency in colon and rectum of both adult and neonate. Further, there was no difference in contractile tension between colon and rectum of adult rats at different temperature. Therefore, the temperature sensitivity of adult colon and rectum appeared to be similar in response to changing temperature. This was not the case with neonate rats. In neonate rats, contractile tension was more in rectum as compared to colon in response to the lowering temperature. Thus, the rectum of neonate seemed to be more responsive to temperature change. This sensitivity of rectum disappeared in adult and was equal to colon. On the whole it may be speculated that sensitivity of rectum in neonate is blunted during development. An increase in contractile tension was observed in different part of adult rat gastrointestinal tract on lowering bath temperature (Mustafa and Thulesius, 2001; Dong et al., 2010). These reports support the present result.

Lowering the temperature of the organ bath has been known to abolish rhythmic activity of gut smooth muscle as evidences by decrease in contractile frequency was observed when bath temperature decreases from 37-15 C in colon and rectum of adult and neonate rats. Such response on frequency has been also shown earlier in dog, mice and rat (Milton and Smith, 1956; Studier et al., 1975; Mustafa and Thulesius, 2001) and consistent with the present finding. The present experiment also detected a higher frequency of rectum as compared to colon at normal body temperature (37 C). However, at lower temperature (20-15 C), the difference in frequency got lost (figure 3). It is further interesting to note that this kind of temperature-dependent difference between colon and rectum contractile frequency was absent in neonate. These suggest that during development there is change in temperature sensitivity that determines contractile frequency.

As the maximum contractile response was observed between $15 \mathrm{C}$ and $20 \mathrm{C}, 20 \mathrm{C}$ was used for further experiments with antagonists to understand the mechanism of cold $(20 \mathrm{C})$ induced contractile response. In the present investigation pre-application of atropine, a muscarinic blocker failed to block the contractile response produced at $20 \mathrm{C}$ suggesting contractions mediated via non cholinergic mechanism or contractile tension to lowering temperature did not depend on a neural mechanism nor the release of neurotransmitters as reported in rat gut (Mustafa and Thulesius, 2001). Similar type of result to atropine was also reported in rat proximal and distal colon (Dong et al., 2010). 
Whereas, diltiazem $(10 \mu \mathrm{M})$ which is a L-type calcium channel blocker, inhibited contractile tension in adult rat rectum only and no such response was observed in case of neonate colon and rectum. On the other hand nifedipine ( 1 and $10 \mu \mathrm{M}$ ), also a L-type calcium channel blocker failed to block the effect of low temperature on both colon and rectum of neonate as well as adult rats. Whereas, in another study by Dong et al. (2010) on rat proximal and distal colon, nifedipine showed to block the effect of low temperature $(17 \mathrm{C})$. Thus, the contractile response in the present study appeared to involve diltiazem sensitive L-type $\mathrm{Ca}^{2+}$ channel in adult rectum only. In addition to this mechanism, contractile response to low temperature might also involved transient receptor potential A1 (TRPA1), a member of transient receptor potential channel family as suggested earlier (Dong et al., 2010). TRPA1 is known to activate by noxious cold stimuli (McKemy et al., 2002; Karashima et al., 2009). TRPA1 agonist i.e. allyl isothiocynate pretreatment inhibited cold-induced contractions and suggested contraction of gut via $\mathrm{PLC} / \mathrm{IP}_{3} / \mathrm{Ca}^{2+}$ pathway or by regulation of intracellular $\mathrm{Ca}^{2+}$ (Sabeur, 1996; Mustafa and Thulesius, 2001; Dong et al., 2010).

\section{Conclusion}

It is concluded that contractile tension and frequency in colon and rectum are temperature sensitive and involves diltiazem sensitive L-type $\mathrm{Ca}^{2+}$ channel in adult rectum. Further, neonate rectum is more responsive to cold-induced contraction by a mechanism not involving $\mathrm{Ca}^{2+}$ channels. The temperatureinduced changes in contractile response in colon and rectum clearly suggest that there is change in the concerned mechanism during gut development process.

\section{Acknowledgement}

Authors are thankful to UGC (New Delhi, India) for financial assistance.

\section{References}

Dong Y, Shi HL, Shi JR and Wu DZ. (2010) Transient receptor potential $\mathrm{A} 1$ is involved in cold-induced contraction in the isolated rat colon smooth muscle. Acta Physiologica Sinica 62: 349-356.

Karashimaa Y, Talaveraa K, Wouter E, Annelies J, Kelvin Y Kwanc, Rudi V, Bernd N and Thomas V. (2009) TRPA1 acts as a cold sensor in vitro and in vivo. Proc. Nat. Acad. Sci. USA 106: 1273-1278.

McKemy David D, Werner MN and David J. (2002) Identification of a cold receptor reveals a general role for TRP channels in thermosensation. Nature 416: 52-58.

Milton GW and Smith AWM. (I956) The pacemaking area of the duodenum. J. Physiol. I32: 100-114.

Mustafa SM and Thulesius O. (2001) Cooling-induced gastrointestinal smooth muscle contractions in the rat. Fundam. Clin. Pharmacol. 15: 349-354.

Sabeur G. (1996) Effect of temperature on the contractile response of isolated rat small intestine to acetylcholine and $\mathrm{KCl}$ : calcium dependence. Arch Physiol. Biochem. 104: 220-228.

Studier EH, Behrend TA and Freed AU. (1976) Effect of temperature on intrinsic intestinal motility in a hibernator. J. Thermal Biol. l: 149-151.

Takeuchi K, Nishiwaki H, Niida H and Okabe S. (1990) Body temperature-dependent action of baclofen in rat stomach. Relation to acid secretion and ulcerogenicity. Digest. Diseases Sci. 35: 458-466.

Theodor V. Burdyga and Susan W. (2002) On the mechanisms whereby temperature affects excitation-contraction coupling in smooth muscle. J. Gen. Physiol. 119: 93-104.

Yano S, Matsukura H, Shibata M and Harada M. (1982) Stress procedures lowering body temperature augment gastric motility by increasing the sensitivity to acetylcholine in rats. J. Pharmacobiodyn. 5:582-92. 